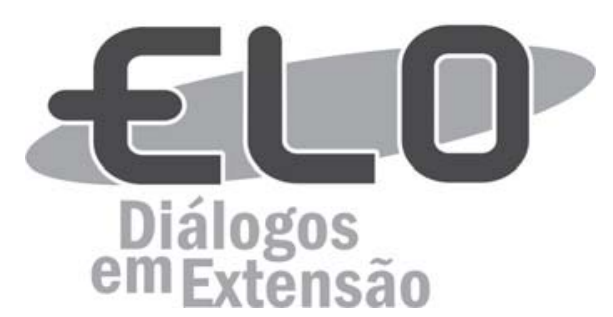

\title{
O impacto de ações de divulgação da neurociência junto a uma comunidade escolar de Uruguaiana/RS
}

\author{
Mayúme de Freitas Fantti ${ }^{1}$, Liane da Silva de Vargas², \\ Ben-Hur Souto das Neves ${ }^{3}$, Pâmela Billig Mello-Carpes ${ }^{4}$
}

\begin{abstract}
Resumo: Já é reconhecida a importância dos conhecimentos da neurociência para a sociedade. Estes conhecimentos, por ficarem restritos ao meio científico, muitas vezes não chegam à população em geral, $e$ tampouco à escola, onde o entendimen to do cérebro poderia contribuir para os processos de ensino-aprendizagem. Esta pouca divulgação científica acaba propiciando a disseminação de neuromitos, entendimentos equivocados acerca do funcionamento do cérebro, os quais podem ser prejudiciais. O programa POPNEURO busca promover ações de divulgação e popularização da neurociência. Neste estudo relatamos o impacto de um conjunto de ações, denominadas Neuroblitzes, que são visitas semanais a uma turma de escolares da Educação Básica nas quais temas de neurociência são abordados em atividades teórico-práticas. Participaram das ações aqui relatadas, realizadas em 2018, 28 escolares do $5^{\circ}$ ano do Ensino Fundamental da Escola Municipal Cabo Luiz Quevedo, do município de Uruguaiana/RS. Para avaliar o impacto das intervenções, um questionário sobre os temas abordados foi aplicado antes, e outro após as atividades. Os resultados demonstram que ações propostas aumentaram o conhecimento de estudantes da Educação Básica sobre o cérebro e o sistema nervoso, como no questionamento sobre os constituintes de um neurônio, no qual, após as intervenções, mais de $40 \%$ acertou todos os constituintes de um neurônio. Também em relação à Universidade, onde antes das ações 68,18\% afirmaram conhecer a UNIPAMPA, e, após as intervenções, este percentual subiu para 87,5\%. Adicionalmente, os alunos gostaram das atividades, considerando-as divertidas e conseguindo estabelecer relação com o que foi destacado nas aulas, com o seu cotidiano.
\end{abstract}

Palavras-chave: Extensão Universitária. Neuroeducação. Neuromitos. Sistema Nervoso. Casos Clínicos. Área Temática: Saúde. Educação.

\section{The impact of neuroscience dissemination actions with a school community in Uruguaiana/RS/Brazil}

Abstract: The importance of neuroscience knowledge to society is already recognized. This knowledge, because it is restricted to the scientific environment, often does not reach the general population, nor to the school, where the understanding of the brain could contribute to the teaching-learning processes. This little scientific divulgation ends up propitiating the dissemination of neuromitos, misunderstandings about the functioning of the brain, which can be harmful. The program POPNEURO seeks to promote actions of dissemination and popularization of neuroscience. In this study we report the impact of a set of actions, called Neuroblitzes. Neuroblitzes are weekly visits to a group of Primary School students in which neuroscience topics are approached in theoreticalpractical activities. Participated in the actions here reported 28 schoolchildren of the 5 th year of Elementary School of the Municipal School Cabo Luiz Quevedo of the municipality of Uruguaiana / RS. The actions were carried out in 2018. To evaluate the impact of the interventions, a questionnaire on the topics addressed was

\footnotetext{
${ }^{1}$ Acadêmica do curso de Fisioterapia da Universidade Federal do Pampa - UNIPAMPA, Uruguaiana -RS, Brasil

${ }^{2}$ Professora da Universidade Federal do Pampa - UNIPAMPA, Uruguaiana -RS, Brasil

${ }^{3}$ Mestrando do Programa de Pós Graduação em Bioquímica da Universidade Federal do Pampa - UNIPAMPA, Uruguaiana -RS, Brasil

${ }^{4}$ Professora da Universidade Federal do Pampa - UNIPAMPA, Uruguaiana -RS, Brasil. Autora correspondente: Laboratório de Neuroquímica, Universidade

Federal do Pampa, BR 472, km 592, CEP 97500-970, Cx postal 118, Uruguaiana/RS/Brasil; +55 (55) 996612454; pamelacarpes@hotmail.com
} 
applied before and after the activities. The results demonstrate that the proposed actions increased the knowledge of Basic Education students about the brain and the nervous system, as in the questioning about the constituents of a neuron where, after the interventions, more than $40 \%$ hit all the constituents of a neuron. Also in relation to the University where before the actions 68.18\% stated that they knew UNIPAMPA, and, after the interventions, this percentage rose to $87.5 \%$. In addition, the students liked the activities, considering them fun and managing to establish a relationship with what was highlighted in class, with their daily life.

Keywords: University Extension. Neuroeducation. Neuroscience. Brain. Scientific Communication. Education.

\section{El impacto de acciones de divulgación de la neurociencia junto a una comunidad escolar de Uruguaiana/RS/Brazil}

Resumen: La importancia del conocimiento de la neurociencia para la sociedad ya está reconocida. Este conocimiento, debido a que está restringido al entorno científico, a menudo no llega a la población en general, ni a la escuela, donde la comprensión del cerebro podría contribuir a los procesos de enseñanza-aprendizaje. Esta pequeña divulgación científica termina propiciando la diseminación de neuromitos, malentendidos sobre el funcionamiento del cerebro, que pueden ser perjudiciales. El programa POPNEURO busca promover acciones de difusión y divulgación de la neurociencia. En este estudio reportamos el impacto de un conjunto de acciones, llamadas Neuroblitzes. Los neuroblitzes son visitas semanales a un grupo de estudiantes de primaria en los que se abordan temas de neurociencia en actividades teórico-prácticas. Participaron en las acciones que aquí se reportaron 28 niños escolares de $5^{\circ}$ año de la Escuela Primaria de la Escuela Municipal Cabo Luiz Quevedo del municipio de Uruguaiana / RS. Las acciones se llevaron a cabo en 2018. Para evaluar el impacto de las intervenciones, se aplicó un cuestionario sobre los temas abordados antes y después de las actividades. Los resultados demuestran que las acciones propuestas aumentaron el conocimiento de los estudiantes de educación básica sobre el cerebro y el sistema nervioso, como en el cuestionamiento sobre los constituyentes de una neurona donde, después de las intervenciones, más del $40 \%$ afecta a todos los constituyentes de una neurona. También en relación con la Universidad donde antes de las acciones, el 68.18\% declaró que conocía UNIPAMPA y, luego de las intervenciones, este porcentaje aumentó a 87.5\%. Además, a los estudiantes les gustaron las actividades, considerándolas divertidas y logrando establecer una relación con lo que se destacó en la clase, con su vida diaria.

Palabras clave: Extensión Universitaria. Neuroeducación. Neuromitos. Sistema Nervoso. Casos Clínicos.

\section{Introdução}

A neurociência pode ser compreendida como o estudo do Sistema Nervoso (SN). Com sua complexa abrangência, ela pode ser subdividida em diversas subáreas, tais como neuroanatomia, neurofisiologia, neuropsicologia, neurociência comportamental, neurociência cognitiva, entre outras. Estas subdivisões compreendem pesquisas em diferentes níveis, que vão desde processos neurobiológicos básicos, em níveis moleculares, até processos comportamentais mais complexos (LENT, 2011).

Embora de reconhecida complexidade, o estudo da neurociência pode explicar aspectos estruturais/ anatômicos do SN, tais como a divisão em sistema nervoso central e periférico; aspectos funcionais, como o controle exercido pelo sistema nervoso somático e autônomo; bem como fenômenos cotidianos, como a atenção que mantemos em determinados estímulos, enquanto em outros não; a capacidade de aprender algumas coisas e outras não; o sono que sentimos; a influência de hábitos saudáveis sobre a nossa capacidade de aprendizagem etc. (FILIPIN et al., 2016). Tomados em conjunto, esses fenômenos compõem a base para o entendimento de como o cérebro aprende, o que é de fundamental importância para a educação.

No entanto, embora a neurociência esteja presente no nosso cotidiano, seu estudo ainda é pouco acessível para a sociedade em geral, incluindo os profissionais da educação. Isso se deve, em grande parte, à linguagem usada em artigos científicos de áreas especializadas, além da pouca presença da neurociência na formação inicial dos professores e no currículo escolar (GERMANO, 2007). Assim, é importante que as descobertas neurocientíficas sejam divulgadas em uma linguagem acessível tanto no ambiente acadêmico, quanto para a população em geral.

Quando feita de forma rigorosa e metódica, a divulgação de conhecimentos da neurociência pode garantir e/ou contribuir com a manutenção e/ou melhora da saúde mental, qualidade de vida e, 
principalmente, com a qualificação da educação (VARGAS et al., 2011). A relação entre neurociência e educação permite explorar as potencialidades do SN de forma criativa e autônoma e, ainda, sugerir intervenções significativas, baseadas em evidências, para a melhora do aprendizado escolar (COSENZA; GUERRA, 2011). Através de atividades de extensão universitária em ambiente escolar, junto a estudantes, essa divulgação pode ser feita desde os anos iniciais do ensino fundamental, até os anos finais do ensino médio, por meio do uso de estratégias didáticas adequadas à idade e escolaridade dos estudantes.

Embora pouco divulgados por neurocientistas, os conceitos acerca do funcionamento do cérebro e do SN sempre geraram muita curiosidade na população em geral, fazendo com que estes conhecimentos frequentemente sejam abordados pela mídia, muitas vezes de forma equivocada, gerando os chamados neuromitos (HOWARD-JONES, 2014). O neuromito surge quando conceitos da neurociência são mal interpretados, seja por um entendimento equivocado, ou por uma citação ou leitura errada de pesquisas neurocientíficas (OECD, 2002). Estes neuromitos, quando divulgados e propagados, contribuem para desinformação e distorção de conceitos científicos, o que favorece a permanência desses equívocos sobre o cérebro (HOWARD-JONES, 2014). Desmistificar os neuromitos é fundamental para que a população possa fazer escolhas conscientes e condizentes com a saúde e bem-estar do SN.

Assim, é importante a divulgação da neurociência, por intermédio de ações de extensão universitária junto à escola, uma vez que a neurociência está pouco presente na formação inicial dos professores e no currículo escolar proposto aos estudantes (GERMANO, 2007). As funções do cérebro, que envolvem desde nossas complexas funções cognitivas até o controle de funções simples de nosso dia a dia, como relações interpessoais, necessidades fisiológicas de alimentação, sono etc., dependem do adequado funcionamento do $\mathrm{SN}$, da mesma forma que os processos de aprendizagem e educação (GUERRA, 2010). Dessa forma, fica clara a relação da neurociência com a educação, e o quão relevante é essa aproximação entre as duas áreas. Assim, a divulgação da neurociência, por meio da extensão universitária, contribui para educação e estabelece esse vínculo, bem como populariza essa ciência na comunidade escolar e na sociedade, de modo geral (PAPADATOU, 2017).

\section{Objetivos}

Este estudo tem como objetivo principal relatar o impacto de ações de divulgação da neurociência e a importância da aproximação acadêmica com a comunidade escolar por meio de atividades de extensão universitária realizadas junto a estudantes do $5^{\circ}$ ano do ensino fundamental de uma escola de Educação Básica do município de Uruguaiana/RS/Brasil.

\section{Metodologia}

Participaram deste estudo estudantes do $5^{\circ}$ ano do Ensino Fundamental da Escola Municipal Cabo Luiz Quevedo, da cidade de Uruguaiana/RS/Brasil. A escolha da escola considerou o Índice de Desenvolvimento da Educação Básica (IDEB). Para o ano 2017, a meta do IDEB para a escola era de 5,9. Contudo, para o mesmo ano, a nota atingida foi de 5,4. Além disso, foram considerados os dados fornecidos pela Secretaria Municipal da Educação (SEMED) de Uruguaiana/RS. A instituição escolhida, que oferta ensino do $1^{\circ}$ ao $9^{\circ}$ ano do Ensino Fundamental, tem 933 alunos matriculados. Quanto às taxas de rendimento escolar, a SEMED informou que, em 2017, o índice de reprovação da escola foi de 7,6\%, e a evasão foi 0,6\%. Já os dados do Censo de 2016 (qedu.gov.br) apresentam que a taxa total de reprovação nos finais do Ensino Fundamental foi de 9,1\% (34 estudantes reprovados). Salienta-se, conforme informado no site do Qeduc.gov.br, que nas taxas acima de 5\% a "situação indica a necessidade de definir estratégias para conter o avanço da evasão escolar" e, acrescenta-se, "da reprovação". A escolha da turma do quinto ano foi conforme critérios da direção da escola.

As ações de intervenção foram vinculadas ao programa de extensão universitária "POPNEURO: Ações para divulgação e popularização da neurociência", promovido pelo Grupo de Pesquisa em Fisiologia (GPFis), da Universidade Federal do Pampa (UNIPAMPA) e foram realizadas em 2018. A equipe do programa é multidisciplinar, sendo formada por estudantes de graduação de diferentes cursos da área da saúde e de licenciaturas e por estudantes de pós-graduação do curso de especialização em Neurociência Aplicada à Educação, e dos Programas de Pós-Graduação em Bioquímica e Ciências Fisiológicas da UNIPAMPA. Além disso, o programa conta com a coordenação 
e colaboração de docentes pesquisadores nas áreas de Anatomia, Educação, Fisiologia, Neurociências e Neuromecânica. Todos os membros da equipe são previamente treinados para a realização das atividades. Este projeto foi aprovado pelo Comitê de Etica em Pesquisa da UNIPAMPA (parecer $n$. 3.138.705); os participantes e responsáveis concordaram com a participação e uso de imagens para divulgação do projeto. No total, 28 estudantes participaram das atividades propostas.

O programa POPNEURO promove uma série de ações extensionistas de divulgação e popularização da neurociência. Entre essas ações, estão as "Neuroblitzes" aqui relatadas. Elas foram realizadas ao longo de um ano acadêmico, por meio de uma visita semanal, com duração de no máximo $1 \mathrm{~h}$, realizadas com uma turma de estudantes da Educação Básica para abordar tópicos de neurociência. As visitas envolveram atividades teóricas e, prioritariamente, atividades práticas, as quais tiveram os alunos como protagonistas. A infraestrutura necessária para as atividades diferenciou-se a cada aula dependendo da atividade, foi realizada tanto no espaço interno, quanto no pátio da escola, em horário escolar, de acordo com o acordado com a escola. Os materiais necessários foram fornecidos pela equipe do projeto, sendo disponibilizado um caderno de anotações personalizado do projeto POPNEURO para que os alunos pudessem fazer seus registros nele.

Antes de iniciadas as ações, a equipe do programa POPNEURO planejou as intervenções. Elas foram propostas e apresentadas pelos membros da equipe em reuniões que aconteceram uma vez por semana. Nessas reuniões, além da apresentação da proposta de atividade e sua discussão e adequação, foram discutidos artigos para embasamento científico sobre os temas trabalhados. Neste estudo, propôsse a realização de 15 "Neuroblitzes" ao longo do ano de 2018, organizadas em cinco temas principais (módulos), brevemente descritos abaixo:

\section{Módulo 1 - Introdução à neurociência}

Neste módulo as ações foram planejadas com a intenção de apresentar os aspectos básicos do $\mathrm{SN}$, gerando interesse dos alunos acerca do tema e motivando-os a pensar na presença da neurociência em seu cotidiano. O módulo foi organizado em quatro semanas. Na semana 1, a visita possibilitou que os alunos estabelecessem conhecimentos iniciais sobre neurociência, introduzindo ao tema do projeto. Foi apresentado o programa POPNEURO e a UNIPAMPA, destacando as formas de ingresso no Ensino Superior. Nesta aula, os alunos receberam um caderno do projeto POPNEURO, para apontamentos livres sobre as intervenções. Na semana 2, por meio de dinâmicas envolvendo a montagem de peças anatômicas do cérebro em grupo e a confecção de um capacete simulando o cérebro, foram apresentados conteúdos que envolveram a anatomia do SN Central e Periférico (Figura 1 D, E e F). Na semana 3, apresentamos aos estudantes as diferentes células do SN em uma peça teatral cujos personagens eram essas células. Na quarta semana foi abordada a comunicação entre as células do SN, necessária para realizar as suas funções; informações como excitabilidade celular, neurotransmissão e contração muscular foram abordadas de forma lúdica nessa aula.

\section{Módulo 2 - Neuromitos}

Neste módulo as ações foram planejadas para discutir ideias equivocadas ou mal interpretadas acerca de neurociência que são comumente consideradas verdades entre a população em geral: os chamados neuromitos (OECD, 2002). O módulo foi organizado em duas semanas. Na primeira semana, mais do que abordar o que são neuromitos, a atividade foi planejada para que os alunos reconhecessem a importância da divulgação científica. Abordamos dois neuromitos muitos comuns, a ideia de que "usamos apenas $10 \%$ do nosso cérebro" e a de que pode existir uma "pílula da inteligência". As atividades permitiram relacionar as funções dos lobos cerebrais e sua interdependência, e a percepção de que utilizamos $100 \%$ da capacidade do nosso cérebro o tempo todo. Ainda, foi discutido que "pílulas da inteligência" não existem, mas que existem sim meios que nos ajudam a melhorar a saúde do nosso cérebro, melhorando nossa capacidade cognitiva, como hábitos de alimentação saudáveis, prática de exercícios físicos etc. (Figura 1 A, B e C). Na segunda aula, o tema neuroplasticidade foi discutido no conteúdo que envolve os chamados períodos de aprendizagem, anos da infância nos quais teríamos a capacidade de aprender coisas novas. Destacouse que o cérebro está sempre pronto a aprender, e que se modifica a partir das experiências durante toda a vida, independentemente da idade. 


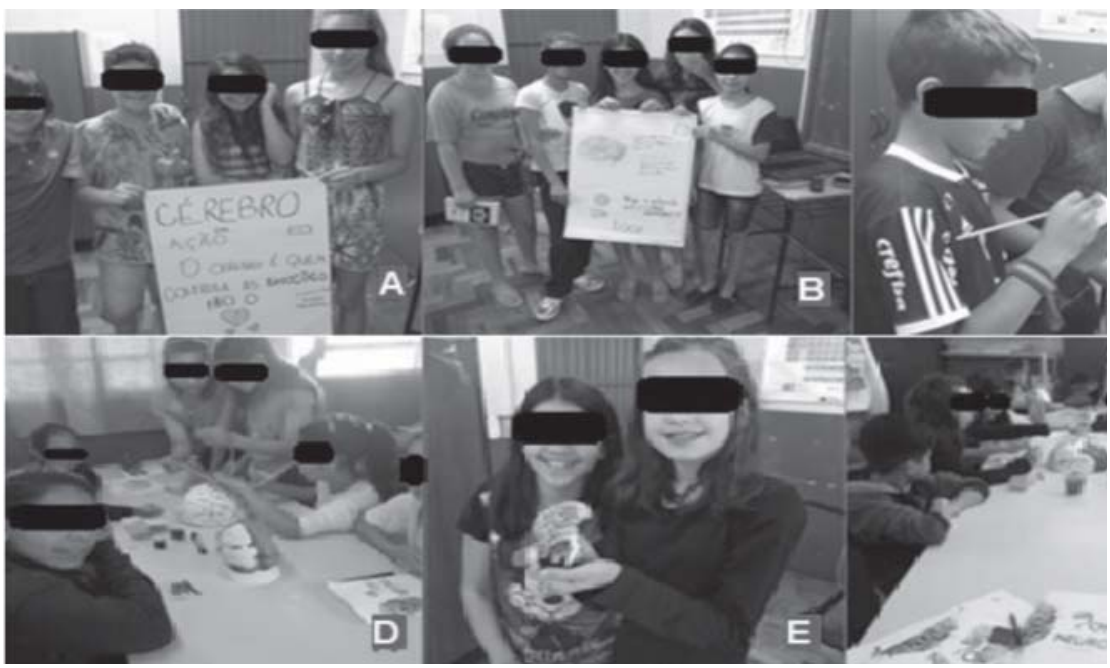

Figura 1. Alunos realizando atividades práticas. Nas imagens A, B e C, práticas relacionadas ao módulo Neuromitos; e, nas imagens D, E e F, atividades desenvolvidas na aula de neuroanatomia, do módulo Introdução à Neurociência.

Fonte: Os autores (2018)

\section{Módulo 3 - Casos Clínicos}

Neste módulo utilizamos casos clínicos de situações comuns ou conhecidas na neurociência para trabalhar temas relevantes para a saúde. O módulo foi organizado em quatro semanas. Na primeira semana trabalhamos com dois casos clássicos da neurociência: dos pacientes HM e Gage, que permitem a abordagem de conteúdos de memória e funções executivas, respectivamente. Na semana seguinte abordamos duas patologias neurodegenerativas bastante comuns: o Acidente Vascular Cerebral (AVC) e a Doença de Parkinson. Na terceira semana do módulo abordamos o autismo e a Síndrome de Down, e temas como bullying e inclusão na sala de aula. Na última semana abordamos o tema neurotraumas, abordando as possíveis consequências de acidentes automobilísticos no caso de traumas na cabeça, e os riscos de chacoalhar um bebê recém-nascido.

\section{Módulo 4 - Saúde e SN}

Neste módulo as atividades foram planejadas para discutir atitudes que contribuem para a saúde e bem-estar do SN. O módulo foi organizado ao longo de três semanas. Na primeira semana foram discutidos cuidados com a saúde e hábitos de vida saudáveis, e como estes podem contribuir para o bom funcionamento do nosso SN, enfatizando a relação entre sono, exercício físico e alimentação com a aprendizagem. Na semana seguinte abordamos como as drogas agem sobre o SN e porque elas podem gerar comportamentos de vício e dependência química. Por fim, na última semana deste módulo, uma atividade enigmática e estimulante procurou abordar o tema raciocínio lógico, com charadas e questões importantes para valorização da leitura. Foi possível discutir funções como linguagem e funções executivas.

\section{Módulo 5 - Ciência}

Neste último módulo abordou-se a neurociência e a pesquisa científica. O módulo foi organizado em duas semanas. Na primeira semana discutimos como se faz pesquisa científica em neurociência e a importância do uso de modelos animais em alguns tipos de pesquisa, as limitações e os aspectos éticos. Na última semana buscou-se estimular os alunos e alunas que se interessassem pela ciência para que visualizassem que eles também podem ser cientistas, desmistificando a imagem deste profissional. A participação feminina (estereótipo de gênero) na ciência também foi discutida.

Em cada tema foi realizada uma breve abordagem teórica inicial, com utilização de projetor e slides, seguida de atividades práticas relacionadas ao tema (brincadeiras, jogos, teatro, experimentação, etc.). Para aulas práticas os materiais utilizados foram diversos, incluindo materiais de papelaria, peças anatômicas sintéticas, microscópios etc. As aulas baseiam-se na proposta do método construtivista e explorando a maneira lúdica de ensinar. Métodos de ensino mais lúdicos, além de serem alegres e 
estimulantes, tornam a prática do ensino bastante dinâmica, e isso enriquece a troca de conhecimentos, despertando a curiosidade para o tema introduzido (OLIVEIRA, 2015).

Para a coleta de dados foi utilizado um questionário construído pelos autores, composto por duas questões de múltipla escolha e sete afirmações para as quais os alunos deveriam afirmar se concordam ou não (Tabela 1). O questionário foi aplicado no início e no final das intervenções. Ambos abordaram aspectos/conteúdos relacionados à neurociência, e aos tópicos trabalhados nas intervenções. $\mathrm{O}$ questionário final contou, ainda, com questões que procuraram verificar a opinião dos participantes sobre as intervenções realizadas: foram apresentadas cinco afirmações com as quais os alunos deveriam expressar seu grau de concordância (Tabela 1). Os questionários foram tabulados e os resultados obtidos são apresentados na forma de percentual.

Tabela 1 - Questionário utilizado para avaliar o impacto das ações de Neuroblitz, aplicado antes e após as intervenções

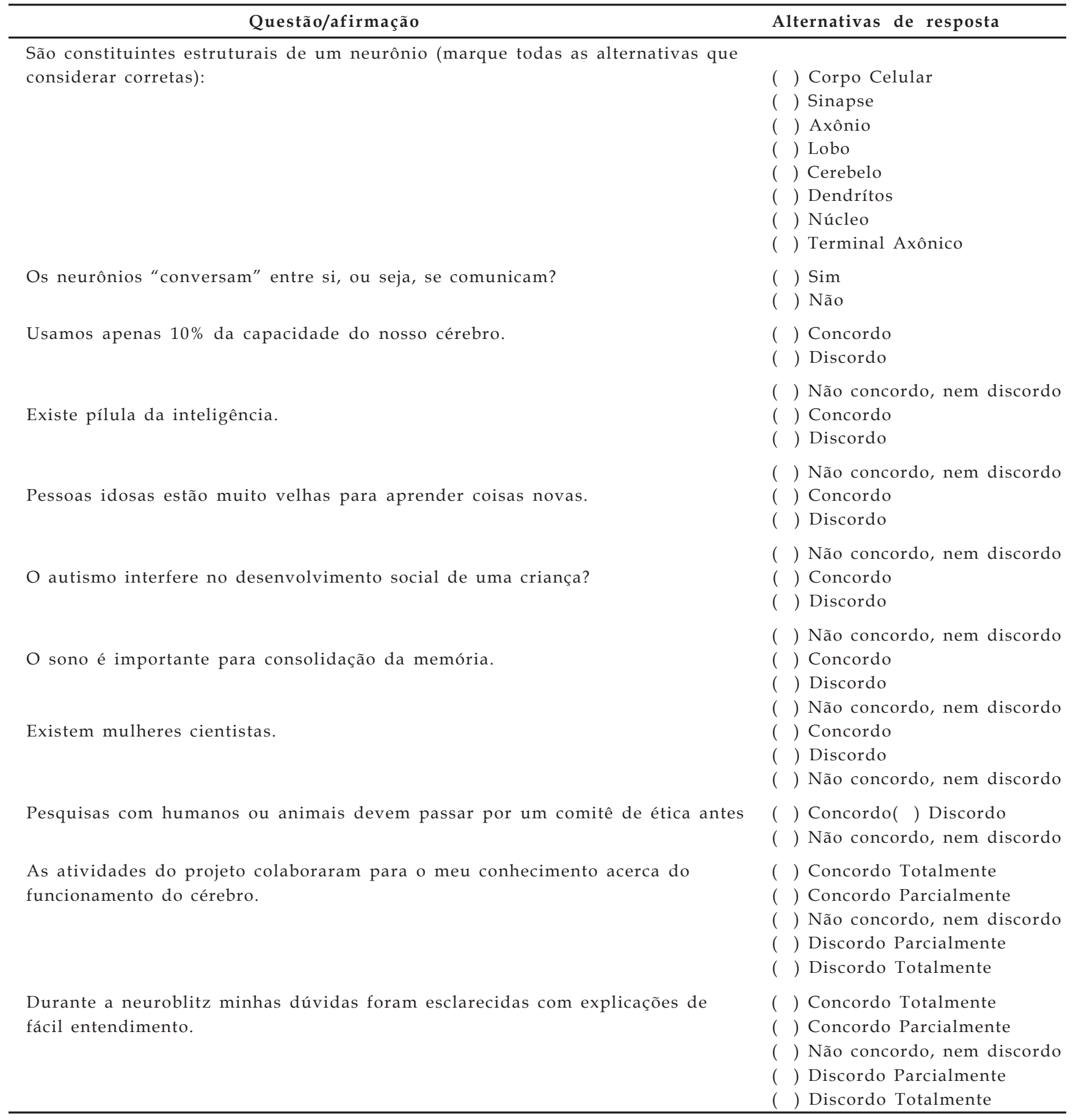

Continua... 
Tabela 1 - Cont.

\begin{tabular}{|c|c|}
\hline Questão/afirmação & Alternativas de resposta \\
\hline $\begin{array}{l}\text { As dinâmicas oferecidas pelo grupo foram divertidas, motivadoras e auxiliaram } \\
\text { no meu entendimento sobre o conteúdo. }\end{array}$ & $\begin{array}{l}\text { ( ) Concordo Totalmente } \\
\text { ( ) Concordo Parcialmente } \\
\text { ( ) Não concordo, nem discordo } \\
\text { ( ) Discordo Parcialmente } \\
\text { ( ) Discordo Totalmente }\end{array}$ \\
\hline $\begin{array}{l}\text { Com as neuroblitzes consegui estabelecer relação da Neurociência com } \\
\text { meu dia-a-dia. }\end{array}$ & $\begin{array}{l}\text { ( ) Concordo Totalmente } \\
\text { ( ) Concordo Parcialmente } \\
\text { ( ) Não concordo, nem discordo } \\
\text { ( ) Discordo Parcialmente } \\
\text { ( ) Discordo Totalmente }\end{array}$ \\
\hline Gostei de ter participado das atividades & $\begin{array}{l}\text { ( ) Concordo Totalmente } \\
\text { ( ) Concordo Parcialmente } \\
\text { ( ) Não concordo, nem discordo } \\
\text { ( ) Discordo Parcialmente } \\
\text { ( ) Discordo Totalmente }\end{array}$ \\
\hline
\end{tabular}

Fonte: Os autores (2018).

Nota: $\left(^{*}\right)$ Questões para avaliação da percepção dos alunos quanto às atividades do programa, incluídas apenas no questionário aplicado após as atividades.

\section{Resultados e Discussão}

Do total de 28 estudantes que participaram das atividades propostas, 22 estavam presentes no primeiro dia de intervenção e responderam ao questionário inicial. Na finalização das ações, que coincide com a data de aplicação do questionário final, 16 alunos estavam presentes e responderam. Neste estudo, demonstramos que houve aumento do conhecimento dos escolares acerca do funcionamento do cérebro após intervenções teórico-práticas que divulgaram a neurociência na escola. Ainda, os alunos afirmaram ter gostado de participar das atividades, as quais foram consideradas divertidas e motivadoras, o que torna possível relacionar a comunidade escolar e a Universidade por intermédio de ações de divulgação científica em um programa de extensão universitária.

Na primeira intervenção, quando questionados se conheciam a UNIPAMPA, $68,18 \%(n=14)$ afirmaram conhecer, e, após as intervenções, este percentual subiu para 87,5\% (n=14) (Figura 3). Através das Neuroblitzes, muitos alunos que não conheciam a Unipampa puderam conhecer a universidade, suas características e sua importância para avanços científicos; embora inicialmente alguns alunos já conhecessem a universidade, o percentual daqueles que não conheciam caiu consideravelmente após as ações (Figura 3). Isso tornou favorável que esse conhecimento prévio seja estabelecido, uma vez que esses alunos residem no mesmo município onde a universidade está instalada, e a partir das ações puderem aproximar-se desse meio acadêmico.
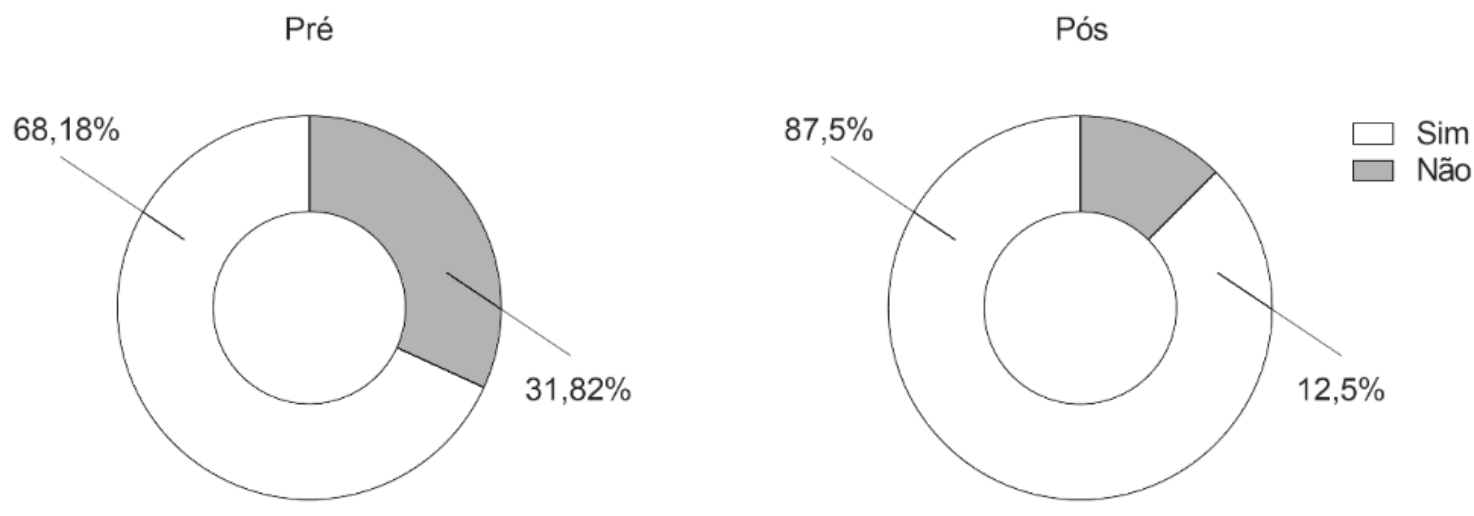

Figura 3 - Porcentagem de alunos que afirmou conhecer a UNIPAMPA antes e após as Neuroblitzes. Fonte: Os autores (2018). 
Quando questionados sobre quais são os constituintes de um neurônio, principal célula do SN, antes das intervenções pouco mais de $20 \%(n=5)$ dos alunos soube identificar o axônio, o corpo celular e o núcleo, e poucos identificaram os dendritos e o terminal axônico como estruturas dessa célula (Figura 4A). Após as atividades semanais, verificamos o aumento do conhecimento dos escolares, visto que mais de $40 \%(\mathrm{n}=8)$ dos alunos marcou todos os constituintes de um neurônio, e embora um percentual de alunos tenha marcado opções incorretas, como lobos e sinapse, talvez por estas estruturas terem sido mencionadas em outras ações, o percentual de alunos que marcou cerebelo, um órgão do SN, como um constituinte dos neurônios, diminuiu (Figura 4A).

A

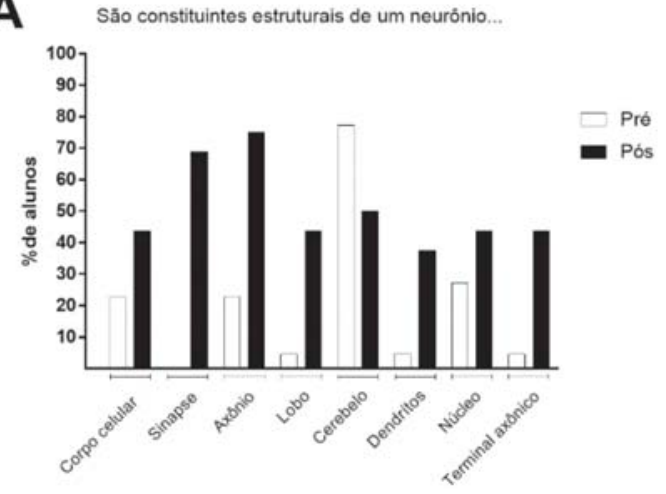

C

Usamos apenas $10 \%$ da capacidade do nosso cérebro.

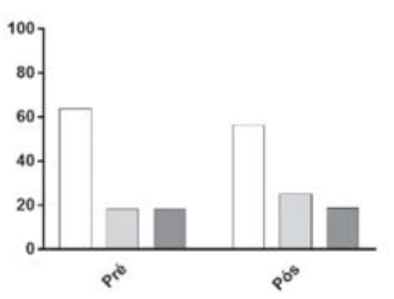

E

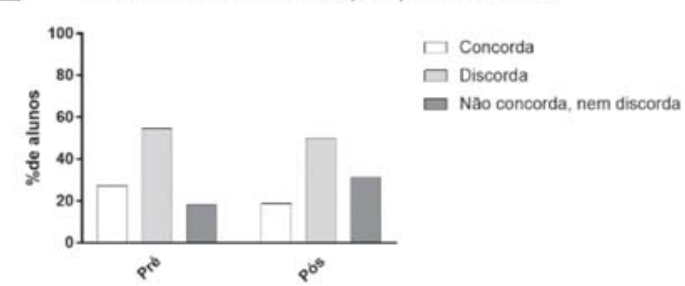

G

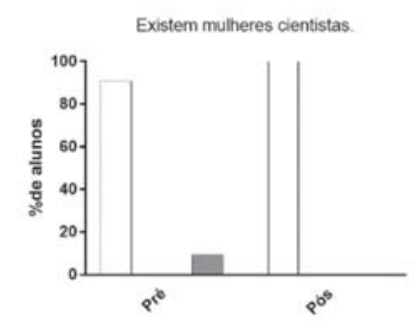

B Os neurônios "conversam" entre si, isto é, se comunicam?

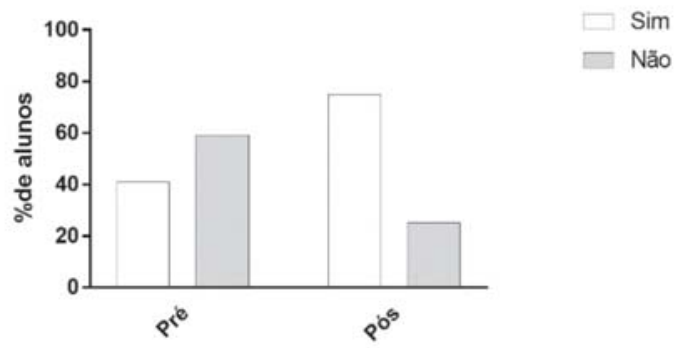

D

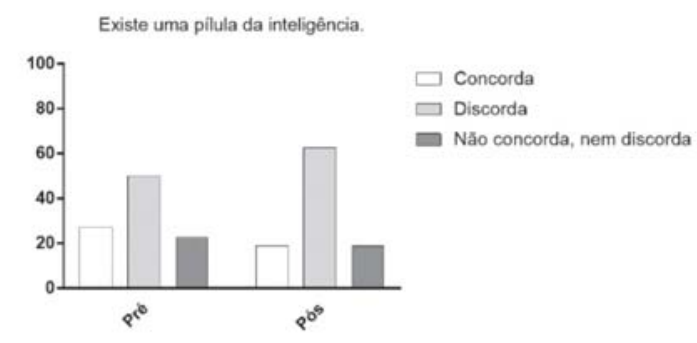

F o autismo intertere no desennvolvimento social de uma criança.

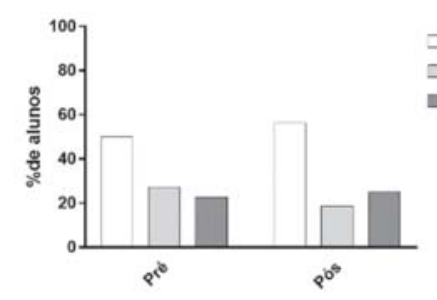

H

Pesquisa com humanos ou animais devem passar por um comité de ética antes de iniciar.

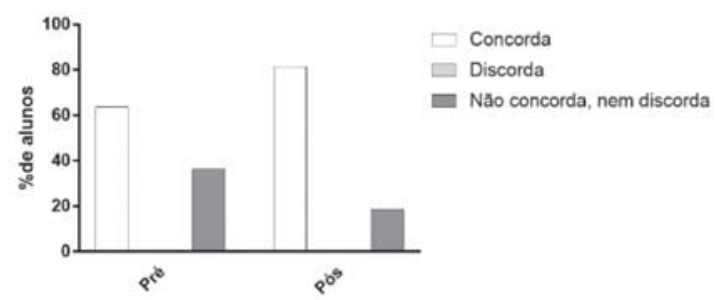

Figura 4 - Respostas às questões do questionário de conhecimentos sobre a neurociência aplicado antes e após as intervenções.

Fonte: Os autores (2018). 
Ao final das ações também pode-se perceber que o percentual do alunos que compreende que os neurônios se comunicam dobrou (Figura 4B). Esse é um dado importante, pois a comunicação celular é a base do funcionamento do SN, e entendendo esse processo em nível celular é possível relacionar como acontecem as funções cerebrais e entender conceitos como a neuroplasticidade. Conforme Cosenza e Guerra (2011, p. 17): "Para compreendermos o funcionamento do cérebro em relação à aprendizagem, que é o nosso objetivo final, é importante que tenhamos um conhecimento básico de como a informação circula por ele".

Além disso, muitos neuromitos foram desmistificados pelas ações: o percentual de alunos que crê que utilizamos somente $10 \%$ do nosso cérebro, que existe uma pílula capaz de nos tornar mais inteligentes e que idosos não são capazes de aprender coisas novas diminuiu (Figura $4 \mathrm{C}$, $4 \mathrm{D}$ e $4 \mathrm{E}$ ). Por mais que essa diminuição não tenha sido tão significativa, podemos considerá-la importante, pois esses são conceitos equivocados fortemente disseminados entre a população (HOWARD-JONES, 2014). Ao trabalhar esses conhecimentos no meio escolar, também contribuímos para que esses estudantes estejam menos suscetíveis a acreditar em outros neuromitos ao longo da vida, pois terão uma visão melhor do que é o cérebro, se tornando mais questionadores e buscando mais informações quando se deparam com afirmações dúbias. Isso é o reflexo da alfabetização em neurociência, que minimiza o impacto da mídia, muitas vezes tendenciosa (DEKKER et al., 2012). Estudo anterior mostra que esse tipo de ação pode ser efetiva a longo prazo: Arce et al. (2017) demonstram que um mês após ações de divulgação de neurociência para escolares, os entendimentos corretos de temas que costumam gerar neuromitos ainda permaneciam.

Adicionalmente, houve entendimento das características do autismo (Figura 4F) mediante atividades que abordaram também a importância da inclusão desses alunos. O aluno é capaz de acolher, desde que entenda a necessidade disso. Sendo a educação um direito de todos, ao conhecer melhor as diferentes condições os estudantes passam a perceber a importância da inclusão nos grupos de sala de aula (CHAVES, 2014), e como esses processos podem ser benéficos não só para os incluídos, mas também para os que incluem.

Por fim, percebeu-se melhor compreensão de como funciona a ciência e de quem pode ser cientista (Figura 4G e 4H). Tão importante quanto divulgar a ciência, é esclarecer para os alunos como ela é feita e quem a faz, quebrando paradigmas relacionados à figura do cientista e à participação de mulheres na ciência.

Ao final das ações, com o objetivo de avaliar a opinião dos alunos acerca das atividades realizadas, foram-lhes apresentadas cinco afirmações com as quais deveriam concordar ou discordar. Observamos que em todas as afirmações houve concordância de pelo menos $75 \%$ dos alunos, demonstrando que eles gostaram das atividades propostas (Figura 5).

Quando se trabalha com crianças e/ou adolescentes, um aspecto importante é a aceitação das atividades. No presente caso foi possível perceber que os alunos, em maioria, afirmaram ter gostado das atividades. Eles consideraram que elas aumentaram seu entendimento sobre o cérebro e que foi possível estabelecer uma relação entre os temas abordados e o cotidiano. Destacamos, ainda, que os alunos consideraram as atividades propostas divertidas e motivadoras. Quando falamos de metodologia pedagógica, principalmente voltada para crianças da Educação Básica, temos que considerar a relação entre as emoções, a motivação e a aprendizagem (OLIVEIRA, 2015). Foi nesse sentido que propomos visitas teórico-práticas que ofertassem diferentes oportunidades de aprendizado aos alunos, nas quais utilizamos métodos lúdicos de ensinoaprendizagem, com uma linguagem adaptada para a faixa-etária, considerando a cultura desses alunos e o conhecimento prévio deles sobre o tema, tornando o ambiente propício para um aprendizado, de forma que fugisse da rotina da sala de aula, mas que, ao mesmo tempo, levasse informações novas e relevantes.

Esse conjunto de resultados demonstra que o impacto das ações de divulgação da neurociência realizadas junto aos estudantes da escola de Educação Básica foi positivo. As ações aumentaram os conhecimentos dos estudantes acerca do funcionamento do cérebro, fornecendo acesso às informações científicas de neurociência de forma clara, divertida e comprometida com a realidade. Além disso, os estudantes gostaram de participar das atividades. 
A

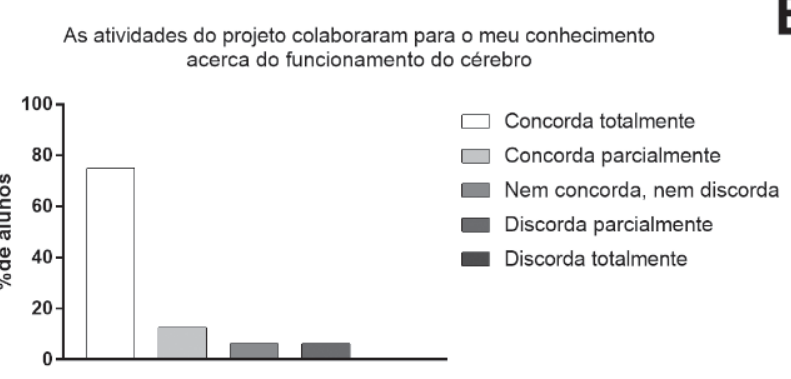

C

As dinâmicas oferecidas pelo grupo foram divertidas, motivadoras e auxiliaram no meu entendimento sobre o conteúdo

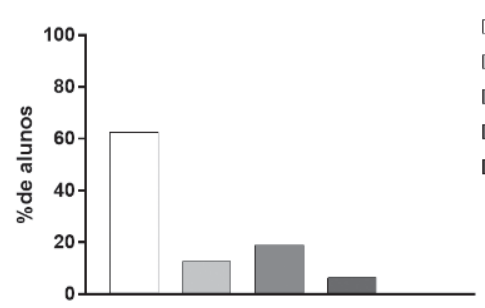

$\square$ Concorda totalmente

$\square$ Concorda parcialmente

Nem concorda, nem discorda

- Discorda parcialmente

- Discorda totalmente
B

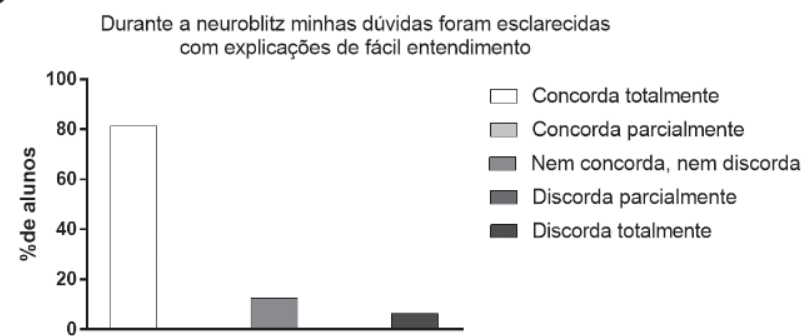

D

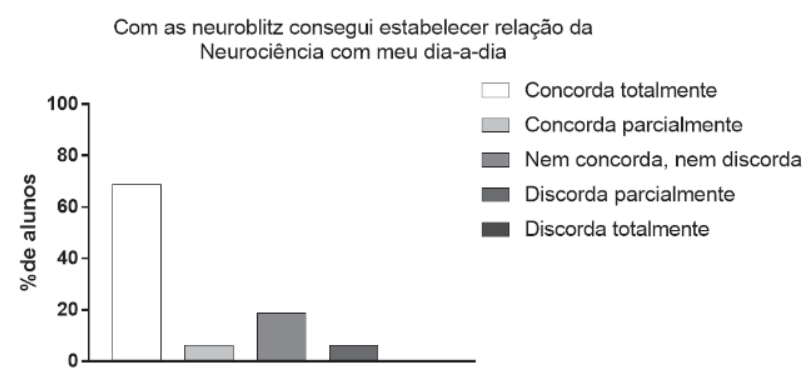

$\mathbf{E}$

Gostei de ter participado das atividades

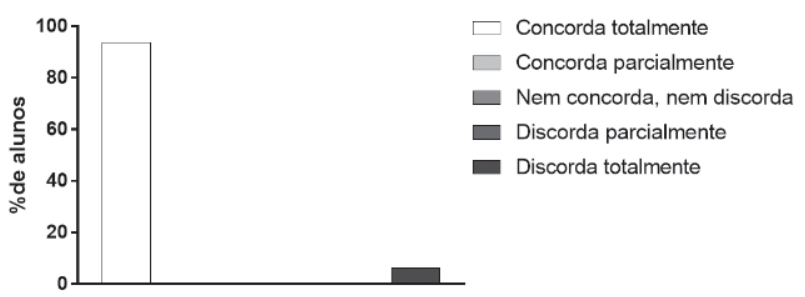

Figura 5 - Opinião dos alunos sobre as ações realizadas.

Fonte: Os autores (2018).

Ainda que a pesquisa em neuroeducação direcione-se mais para os professores, é importante esclarecer aos alunos também sobre essa ciência tão presente no nosso cotidiano. Além dos conhecimentos de neurociência contribuírem para o entendimento de como o cérebro aprende, as ações de comunicação científica também promovem educação em saúde, pois os alunos participantes passam a conhecer melhor seu cérebro, a fisiopatologia de algumas doenças neurodegenerativas e aprendem sobre hábitos que ajudam a manter seu cérebro saudável e prevenir perdas cognitivas e/ou doenças neurodegenerativas. Como nos coloca Vargas et al. (2011, p.32):

(...) sendo a saúde atualmente concebida como um estado dinâmico de bem-estar biológico, psicológico e social, o indivíduo será mais saudável quando mais conhecimento tiver em relação ao seu organismo e as maneiras de preservá-lo e melhorá-lo. Esse objetivo se alcança por meio do acesso a conhecimentos formais que se vinculam ao processo de aprendizagem.

Desse modo, é preciso ajustar o conteúdo científico ao perfil do público-alvo, isso tudo sem correr o risco de perder conteúdo ou promover entendimentos equivocados sobre ele. Assim, os alunos poderão estabelecer os conhecimentos obtidos de maneira adaptada para sua faixa-etária e, ainda, aulas que levem em conta sua cultura e conhecimento prévio formando, dessa forma, uma aprendizagem significativa que valorize a curiosidade dos alunos e sua capacidade de formar opiniões sobre o tema. A verdade é que essa aprendizagem significativa começa desde o momento que os escolares receberam um caderno de anotações personalizado do projeto POPNEURO, onde eles tiveram liberdade para direcionar suas anotações ao que eles entendessem como importante de ser registrado, por estabelecer relações entre o conteúdo proposto e o seu cotidiano, construindo e 
modelando pensamentos por meio do modelo de aprendizagem significativa de Ausubel (PELIZZARI et al., 2002).

Esperamos que este estudo incentive a disseminação de práticas como esta, de maneira responsável. Embora ações de divulgação científica devam ser sempre estimuladas, é importante considerar a rigorosidade metódica e a frequência, assim como a qualidade com que se faz a divulgação científica, deve-se adequar a maneira com que se implanta ela na sociedade, sendo esta uma tarefa difícil e que demanda de uma linguagem adequada para se tratar de conteúdos e linguagens muitas vezes complexas. Essa linguagem científica é mutante, podendo algo que hoje é aceito como verdade não valer daqui há alguns anos mais, e a população precisa ser conscientizada acerca disso (DEKKER et al., 2012). A comunicação científica é necessária, e fortalecer essa relação com o sistema educacional se destaca como fundamental para a construção de uma sociedade cientificamente esclarecida, por consequente mais saudável e com melhor qualidade de vida (BARBA, 2019).

\section{Conclusões}

Os resultados apresentados demonstram que o impacto das ações de extensão universitária por meio de divulgação de neurociência realizadas na escola de Uruguaiana/RS foi positivo, auxiliando no melhor entendimento do funcionamento do cérebro e promovendo a desmistificação de neuromitos. Além disso, os próprios estudantes demonstram gostar desse tipo de atividade, mantendo uma relação positiva na perspectiva de aproximação da comunidade escolar, sociedade e a Universidade.

\section{Agradecimentos}

Os autores do trabalho agradecem à Universidade Federal do Pampa (UNIPAMPA) pelo apoio financeiro a este trabalho no âmbito do Edital PROFEXT e PDA. Agradecem também aos estudantes e à comunidade escolar, bem como a toda a equipe do POPNEURO, envolvidos neste estudo, pela disponibilidade de participarem da pesquisa, direta e indiretamente.

\section{Referências}

ARCE, J. P. S. ; SOUZA, M. M. ; VARGAS, L. S. ; CARPES, P. B. M. . Divulgando a neurociência: ações para desmistificação de neuromitos. REVISTA ELO - DIÁLOGOS EM EXTENSÃO. v. 6, p. 64-73, 2017.

BARBA, MDLP, CASTILLO, JPGD e MASSARANI, L. Envolvimento público na ciência: Mapeando e compreendendo a prática da comunicação científica na América Latina. Anais Da Academia Brasileira de Ciências, p.91, 2019.

CHAVES, C. R. In: Marta Pires Relvas. (Org.). Que cérebro é esse que chegou à escola? As bases neurocientíficas da aprendizagem. 2ed.Rio de Janeiro: WAK Editora, 2014, p.260

COSENZA, R ; GUERRA, L. Neurociência e educação: como o cérebro aprende, 1.ed. Porto Alegre: Artmed, 2011. p. 145.

DEKKER, S, LEE, NC, HOWARD-JONES, P, JOLLES. J. Neuromyths in education : prevalence and predictors of misconceptions among teachers. Fronteiras em Psicologia, 2012.

FILIPIN, G. ; VARGAS, L. ; MELLO-CARPES, P. (Orgs). Popneuro: guia prático de atividades para popularização e divulgação da neurociência. São Paulo: Livro Bits. 2016, p. 11-15.

GERMANO, M; KULESZA, W. Popularização da Ciência: uma revisão conceitual. Caderno Brasileiro de ensino de Física: Florianópolis, SC: Caderno Brasileiro de Ensino de Física, 2007.

GUERRA, L,B. Como as neurociências contribuem para a Educação Escolar?. Revista Fundação Guimarães Rosa (FGR), v.4, p6-9, 2010.

HOWARD-JONES, P. Neuroscience and education: myths and messages. Nature Reviews Neuroscience, v. 15, p. 817-824, 2014.

LENT, R. Cem bilhões de neurônios: conceitos fundamentais de neurociências. São Paulo, SP: Editora Atheneu, 2001 
OLIVEIRA, Gilberto Gonçalves de. A pedagogia da neurociência: ensinando o cérebro e a mente. Curitiba: Appris, 2015. 231 p. 131-133.

Organisation for Economic Co-operation and Development. Understanding the Brain: Towards a New Learning Science. OECD Publications, 2002.

PAPADATOU-PASTOU et al. Brain Knowledge and the Prevalence of Neuromyths among Prospective Teachers in Greece. Frontiers in Psychology, 8:804, 2017.

PELIZZARI, A. et al. Teoria da aprendizagem significativa segundo Ausubel. Revista PEC, Curitiba, v. 2, no 1, p. 37-42, jul. 2001/jul. 2002

VARGAS, G. et al. Neuroeducação: a relação entre Saúde e Educação. Rio de Janeiro: Wak Editora, p. 32,2011

INSTITUTO NACIONAL DE ESTUDOS E PESQUISAS EDUCACIONAIS ANÍSIO TEIXEIRA. Índice de Desenvolvimento da Educação Básica (Ideb). Disponível em < http://idebescola.inep.gov.br/ ideb/escola/dadosEscola/43162347>. Acesso em: 19 de Junho de 2006.

Recebido para publicação em 15/07/2019 e aprovado em 27/08/2019. 